\title{
Perceptions, emotional reactions and needs of adolescent psychiatric inpatients during the COVID- 19 pandemic: A qualitative analysis of in-depth interviews
}

\section{George Giannakopoulos ( $\sim$ ggiannak@uoa.gr)}

Department of Child Psychiatry, School of Medicine, National and Kapodistrian University of Athens, "Aghia Sophia" Children's Hospital https://orcid.org/0000-0001-7427-1776

\section{Savvina Mylona}

National and Kapodistrian University of Athens

Anastasia Zisimopoulou

National and Kapodistrian University of Athens

\section{Maria Belivanaki}

National and Kapodistrian University of Athens

\section{Stella Charitaki}

National and Kapodistrian University of Athens

\section{Gerasimos Kolaitis}

National and Kapodistrian University of Athens

Research article

Keywords: Adolescents, COVID-19, Mental health, Pandemic, Psychiatric inpatient unit

Posted Date: December 2nd, 2020

DOI: https://doi.org/10.21203/rs.3.rs-96637/v2

License: (9) This work is licensed under a Creative Commons Attribution 4.0 International License.

Read Full License

Version of Record: A version of this preprint was published at BMC Psychiatry on July 28th, 2021. See the published version at https://doi.org/10.1186/s12888-021-03378-w. 


\section{Abstract}

Background: The new coronavirus pandemic (COVID-19) has been accompanied by severe psychological pressure on the entire population. However, little is known about how this pandemic could affect the more vulnerable population with severe mental illness.

Aims: To explore adolescent psychiatric inpatients' perceptions, emotional reactions and needs during the first wave of the COVID-19 pandemic.

Methods: Individual in-depth interviews were conducted with nine psychiatric inpatients aged 12-17 years. Through open-ended questions, interviewers initiated five themes: (1) knowledge about the coronavirus pandemic, (2) changes in everyday routine, (3) pandemic-related emotions, (4) helpful thoughts and attitudes and (5) practices for helping adolescents deal with the hardships brought about by the pandemic. A thematic analysis was conducted using line-by-line open coding.

Results: With regard to their knowledge of the impact of the current pandemic, almost all adolescents focused on information about the nature of coronavirus and also on existing crisis management practices. Almost all patients identified predominantly negative changes due to the quarantine state, including restrictions on both social life and personal freedom as well as excessive contact with family members during home isolation. As far as their emotions was concerned, adolescents did acknowledge anxiety about self-harm and harming their loved ones as well as mood swings within the family nucleus; anxiety was also manifested about the unknown and the management of the pandemic in other countries. Avoidance of thought rumination about the coronavirus and its consequences, positive thinking and looking towards the future were reported as constructive strategies for coping with challenging emotions. Additionally, a sense of belongingness (provided that they are not alone and share a common experience) seems to have been playing a pivotal role in the adolescents coping strategies. Trust in the authorities and the community was another quite noteworthy point that emerged during the interviews. Lastly, our findings indicated adolescents' benefit from receiving balanced health messaging coupled with balanced thinking within both their social and family environment.

Conclusions: Enhanced comprehension of possible mediating psychological pathways is needed to help clinicians, researchers and decision-makers to avert the deterioration of mental disorders and overall functioning, as well as additional stress-related disorders.

\section{Background}

The first wave of the new coronavirus pandemic (COVID-19) struck the entire world in the first quarter of 2020 with many countries still struggling to mitigate its magnitude on public health through strict quarantine measures, social distancing and health education strategies [1]. This unprecedented phenomenon is equivalent to a mass disaster. Mass disasters affect individuals, families and entire 
societies and typically result in major physical, psychological and economic consequences. The overall and enduring impact of a mass disaster may disrupt societal networks of children and their families alike, daily life routines and also question all sense of security and cohesion [2]. Under the pandemic threat, society's ability to facilitate the recovery of its members is being dramatically reduced. The situation is aggravated as resources run out and health, education and social welfare facilities are being compromised.

During the ongoing pandemic, morbidity and mortality aside, psychological pressure on the entire population is particularly high. Fear of infection and its outcomes, loneliness, denial as well as anxiety and obsessive-compulsive disorder symptoms, ennui, frustration, irritability, depression and despair may be experienced by a significant part of the population. In addition, the ensuing economic crisis and job loss coupled with social rejection, discrimination and stigma, further jeopardize mental health [3-9].

There are numerous mental health risks for children and adolescents which are pandemic-related [10-14]. During pandemic's acute phase of the pandemic, social distancing, increased pressure on families and minimized access to support services have been observed. Following the pandemic, mental health services have been asked to tackle issues such as economic recession and anxiety, stress and violence exposure $[15,16]$.

Patients with pre-existing severe mental illness are inevitably affected by the aforementioned circumstances, thus experiencing more intense discomfort $[17,18]$. By way of illustration, patients in psychiatric intensive care units may experience the increased threat of a group infection [5], while psychosocial impact on children and adolescents in inpatient psychiatric units is likely to be much more critical [19].

Youths' experiences during a disaster like the current pandemic may usually be classified under three inter-related patterns: (a) disruption of normality, (b) fear due to an either objective or subjective threat to life as well as due to the exposure to fear-provoking information, and (c) mourning following a loss (be it the loss of human life or a sense of meaning as well as of basic trust and/or self-esteem) [2]. However, the inner experience of children and adolescents may be overlooked even by the most sensitive observers. Therefore, clinicians and researchers should take into consideration and evaluate the type, extent, and consequences of children's and adolescents' emotional and behavioral responses. Moreover, children and adolescents with pre-existing severe mental health problems belong to a high-risk group for worse outcomes not least because of disrupted access to mental health services and impairment of the ability for adaptive coping and emotion regulation [15, 20-22].

The aim of our research was to qualitatively investigate, through in-depth interviews, the perceptions, emotional reactions and needs of adolescent psychiatric inpatients amidst COVID-19. The method of indepth interviews offers the opportunity to collect detailed information regarding the comprehension, thoughts and feelings of hospitalized children and adolescents with severe psychiatric disorders. It is hoped that the analysis in question will provide preliminary insight regarding this vulnerable population in the face of an unprecedented disaster. The findings of the present research could enable clinicians, 
researchers and decision-makers develop psychosocial support interventions as well as prevent not merely the deterioration of mental disorders and overall functioning but also additional stress-related disorders.

\section{Methods}

\section{Participants and procedure}

The study included all nine inpatients aged 12-17 years that were treated during the second week of April 2020 in the psychiatric intensive care unit of the Department of Child Psychiatry, School of Medicine, National and Kapodistrian University of Athens, "Aghia Sophia" Children's Hospital. Hence, the study employed a purposive (total population) sampling no saturation point was used to determine the sample size and data collection. Participants 1, 2, 4, 5, and 9 were discharged from hospital to home sheltering and continued their treatment program via Skype (individual supportive psychotherapy, parent counselling and family sessions), while the remaining adolescents (Participants $3,6,7,8$ ) continued inpatient treatment. In this particular setting's modification, the first group of adolescents was interviewed via Skype, while the second group in vivo, in the psychiatric intensive care unit premises. Three patients were treated for anorexia nervosa, one patient for obsessive-compulsive disorder, one patient for major depressive disorder with school refusal, one patient for autism spectrum disorder and conduct disorder, one for adjustment disorder, intentional self-harm and a history of institutional upbringing, whereas two more patients were treated for generalized anxiety disorder, one of which also had enuresis and a history of emotional neglect.

Individual in-depth interviews were conducted with every patient by one female interviewer with appropriate 5-hour training in the interview guide. The second and the third author, who were working as child and adolescent psychiatry trainees in the psychiatric intensive care unit at the time of the study, conducted the interviews. Prior to the final administration, an in-depth interview guide was developed, pilot tested and reviewed. The average duration of each interview was approximately 20 minutes. No one else was present besides the participants and researchers during the interviews. The participants were given a brief description of the current situation pertaining to the COVID-19 pandemic and were subsequently asked to participate in the discussion. They were then asked an open set of questions related to the aims of the present research: (a) What do you know about the new coronavirus? (b) How has your daily life changed due to the coronavirus? (c) How do you feel about what have been happening? (d) Is there anything you can think or do to feel better? (e) Is there anything other individuals can do to help you feel better? The questions were developed for this study and have not previously been published elsewhere.

All of the in-depth interviews were audio-recorded and verbatim transcribed. The transcripts were not returned to participants for comment and/or correction. No field notes were made during the interviews and no repeat interviews were carried out.

\section{Data analysis}


A thematic analysis using line-by-line open coding was conducted to explore the understanding, thoughts and emotions of children and adolescents treated in child and adolescent psychiatric inpatient units [23, 24]. The concepts generated by the raw data were then grouped into conceptual categories. Constructed codes were created from in vivo codes (the exact wording used by participants in the interviews). Constant comparisons for developing themes and seeking data not conforming to each theme were carried out by two researchers independently. At the end of the aforementioned process, no new information was extracted and repetition of information in each of the categories was taken into account. Participants did not provide feedback on the findings.

\section{Results}

The analysis included the themes that were initiated by interviewers through the five open-ended questions: (a) knowledge about coronavirus pandemic, (b) changes in everyday routine due to the pandemic, (c) children's feelings about the pandemic, (d) children's positive thoughts and behaviors, and (e) practices for helping children and adolescents cope with their difficulties related to the pandemic. Each theme is reported below, while examples of their contents are illustrated with participants' quotes.

\section{Knowledge about the coronavirus pandemic}

The concepts that emerged from the raw data and the wider conceptual categories are shown in Table 1. Information about the nature of the coronavirus was the most common answer between the participants concerning the coronavirus pandemic, including the definition of the coronavirus, its worldwide spread rate, the morbidity, the transmission risk/rate as well as the mortality, outcomes and past knowledge about the pandemic and the origins of the virus.

... many people have died from all this... the older ones can easily die... (Participant 1)

...others go through it a little heavily, others more lightly ... I know that mostly older people get sick ... children fortunately go through it very lightly... because, unfortunately, it is very easily transmitted by children to adultsmainly (Participant 8)

A group of participants commented on the existing crisis management practices in Greece and on the existing knowledge about treatment and disease prevention:

In Greece we have handled it quite well, so things are better than in other countries that have been hit harder than us (Participant 1)

... but luckily, here in Greece, there are no many deaths (Participant 8)

... unfortunately, it has not yet been found, at least to the best of my knowledge, either a drug or a vaccine or anything else... we hope to find a vaccine (Participant 2)

Additionally, one participant referred to the psychosocial aftermath of the pandemic: 
The (infected) are afraid of the pandemic and feel anxiety, isolation ... they can't see their beloved ones ... even their friends, their relatives... Very sad indeed (Participant 9)

\section{Changes in everyday life}

The concepts that emerged from the raw data and the wider conceptual categories are shown in Table 2. Initially, three main conceptual categories emerged from the participants' interviews referring to changes in everyday life: positive changes, negative changes and non-important changes.

Regarding the positive changes, only two participants acknowledged certain positive aspects of the pandemic's impact on daily life. Specifically, these aspects comprised of the precautionary measures, the strict measures as well as their necessity and the observed compliance of the population to them. What is more, a sense of transience was recorded. Having said that, all the above is to be considered more as helpful attitudes and beliefs that enable the participants cope with his pandemic-induced stress, rather than through substantially positive aspects of the pandemic.

... unless we consider the general condition worldwide, the strict measures that each country has taken... I understand these measures that have been taken and I believe all this is happening for our own good, so I comply with them like any other citizen... and I believe that all this will pass and we will continue our daily routine as before... (Participant 2)

A second participant viewed the increased contact with beloved ones during home restriction as a positive aspect of the pandemic:

... so, we are all together again... it's been a long time since we'd all been together like that... it was an opportunity to spend some time together... (Participant 1)

Almost all participants mentioned negative changes in everyday life, notably with the newly imposed mandatory protection rules, the curbing of social life as well as the restriction of freedom, while increased contact with beloved ones during home restriction was deemed as a negative aspect too.

Regarding the adaptation of new obligatory protective behaviors, some participants referred to new hygiene rules that must be followed and to a necessary modification of social encountering.

We have to be within distance from each other, we don't hug each other too much... (Participant 3)

Limitations on social life were the most commonly referred to as a negative change. More specifically, nearly all participants referred to the decreased contact with beloved ones, the restrictions on socializing opportunities as well as the indoors confinement and schools' closure.

Another particularly interesting concept that emerged from quite a few participants' responses was that of restriction of personal freedom.

... obviously I can't go out whenever I want to (Participant 1) 
... we have to stay in the house, and we can only go out when... for some important reason, such as going to the pharmacy or to the supermarket, and still one person at a time, etc. (Participant 2)

... we have to send a text message if we need to go out... (Participant 6 )

In addition, one participant recognized a negative aspect related to the increased contact with beloved ones.

... there can sometimes be disagreements or fights about many issues... (Participant 1)

Finally, some participants did not acknowledge any significant changes in everyday life, in view of their pre-existing confinement in the inpatient unit before the onset of the pandemic.

Personally, my daily life has not changed that much (Participant 2)

Ehm... of course in here, ok, things have changed very little ... (Participant 6)

\section{Feelings about the pandemic}

The concepts that emerged from the raw data and the wider conceptual categories are presented in Table 3. The four main conceptual categories that emerged from participants' answers were: negative feelings, positive feelings, ambivalence, and non-important changes in feelings.

Half of the participants expressed worries about possible harm to their loved ones, and one adolescent also considered the psychological impact.

I feel a bit anxious because my mum unfortunately belongs to a vulnerable group ...and I want to know that she is well, all the time... I am very concerned about my grandparents who are old... about the people whom I love and I care about (Participant 8)

I see my brother, who is a student and obviously used to go out very often, being more irritable and getting mad more easily... (Participant 1)

Possible harm to self was referred to a much lesser extent by participants:

... but ok, I can't say I am really worried about contracting it myself... (Participant 1)

Three participants expressed concern about the future and the unknown:

I'm worried about how things will evolve - I mean, how am I to go to school next year ... what will happen with the exams ... all this stresses me out ... I like to know my schedule and not be, like, in a "wait- andsee" situation... (Participant 1)

I feel anxiety about when this thing will come to an end... (Participant 6)

I feel very... uncomfortable... (Participant 7) 
Notably enough, two participants raised concern about the management of the pandemic from a global perspective:

In other countries, cases are either increased daily or are way too many... (Participant 2)

Sadly, there are numerous deaths in some other countries - but not that many here in Greece (Participant 8)

Few participants expressed sadness about the deceased or affected people in general:

I'm sad about all these people who have died, or those who are ill, and we still don't know their outcome... (Participant 2)

I also feel very bad about the other people who used to be free before but now are all homebound... (Participant 8)

However, more expressed their sorrow with regard to the lack of contact with their beloved ones.

I feel a bit sad for not being able to see my dad, because - as a high-risk individual - he is not allowed to get around... (Participant 3)

I also feel sorrow that I do not see my family... (Participant 9)

In some instances, sadness was also associated with anger and ennui:

I feel sadder because I'm mainly bored... (Participant 6)

... I simultaneously feel anger... and maybe sadness... (Participant 9)

Four of the participants emphasised on the feeling of being confined ('trapped', even) and of having been deprived of their freedom. Some of them also said that hospitalisation has been keeping their routine unchanged, whilst others as an aggravating factor of their feelings of confinement and loneliness.

I don't feel very well ... but I manage. Everybody must be feeling like that, like they are 'boxed' in a house (Participant 5)

... It would help me to be out, not in here... to be with my brothers, my family, with the ones I love. ... I cannot do the things I want when I'm shut in here, and I feel like being on my own. (Participant 7)

Several participants expressed optimism:

...well ok, I know that they (family) are cautious, so I am calmer... (Participant 1)

This will not be for too long, now that the temperature is rising and it'll be warm, it will go away (Participant 3) 
...on the other hand, I'm happy to hear on the news that cases are decreasing daily, at least in Greece... I believe everything will be fine... (Participant 2)

Two of the participants reported positive feelings about the lockdown due to the increased amount of time spent with their loved ones.

I enjoy the days spent with my parents, because I know that when we go back to normality my mum will be working work until late, my dad will return to his shifts and all that...; so it was a chance for all of us to be together (Participant 1)

... Yet I feel some joy as well, because in this way I get to have more free time to be with my family and to play several board games ... (Participant 9)

When asked about their feelings about the current situation, some inpatients were in two minds. Mixed feelings aside, two participants expressed non-significant changes in their emotions.

I feel like the other times... ok. I can play with my sisters and watch TV... (Participant 4)

\section{Helpful thoughts and behaviors}

The concepts that emerged from the raw data and the wider conceptual categories are presented in Table 4. Initially, from the participants' interviews about what they could do to feel better two main conceptual categories emerged, namely beneficial thoughts and behaviors.

Some participants referred to the avoidance of ruminating about the pandemic as a helpful mindset during the pandemic. In addition, it appears that focusing on the positive aspects of the current situation, such as the low morbidity- mortality rates in Greece and knowing that their beloved ones are healthy, is a helpful coping mechanism, Another particularly interesting finding was that many participants were helped by focusing on what the future brings - by maintaining, in other words, that this is merely a transient situation; looking forward to resuming their daily routine and seeing the end of restrictions whilst making plans for the future, also seem to be helpful. Moreover, two participants acknowledged the feeling that they are not alone, in the sense that this is a commonly shared experience. This later mode of thinking also seems to be a comforting one that helps them cope with their worries.

....and generally, to not feel as if I'm alone in all this... (Participant 1)

Finally, regarding the thoughts that adolescents found helpful, a crucial factor is that they put trust in both the authorities and in the community. Indeed, many participants positively commented on the solid operation of Greek state's institutions and on the citizens' compliance with the measures. Trust in the scientific community was also reported.

... and I think that Greece has taken preventive measures much earlier, compared to other countries, and [I think] that, at present, we are one of the safest countries (Participant 6) 
... there haven't been many violations (Participant 1)

... we have taken precautions ... and that reassures me... (Participant 6)

... both doctors and experts do the best they can to help people feel safe... (Participant 2)

On the issue of helpful behaviors, few participants admitted that regulating the amount of information received is of paramount importance. More specifically, two participants voice their need to hear positive news about the pandemic so that the former may feel better. One participant, in particular, focused on his need for minimal media information whilst emphasizing their constant need to be kept informed about what has been going on.

I generally don't sit and watch the news all the time... this doesn't help me obviously... I watch very little, just enough so as not to live in a bubble (Participant 1)

Additionally, almost all participants mentioned a need for imaginative leisure activities alongside the need to engage with others through contact with beloved ones as well as by resorting to them for comfort when necessary:

Obviously, talking to my friends and not isolating myself helps; the same goes for talking with my grandparents via Skype as much as I can .... and, when I'm feeling anxious, I visit my parents for support ... I believe in this... (Participant 1)

\section{Practices for helping adolescents deal with the pandemic-related situation}

The concepts that emerged from the raw data and the wider conceptual categories are presented in Table 5. Some participants found information overload to be unhelpful. Instead, adolescents pointed out that it is necessary to calibrate the flow of information received. Nevertheless, they did acknowledge that they need to be kept in the know about the latest developments regarding the pandemic. Additionally, some participants thought that adults' willingness to answer their queries and provide positive updates was beneficial to them.

Obviously [I want the family] to not hide things from me, to not tell me 'everything is fine', because, ok, I wouldn't like to not know what is going on... but I wouldn't like to hear them overanalyzing all this with aimless discussions either (Participant 1 )

A balanced approach and mindset in the adolescent's environment was identified as a helpful strategy by several adolescents, especially by those who focused on their family's positive attitude and avoidance of excessive panic:

... if a family's or the relative's, or the wider environment's general perception is positive and correct, then the child does not worry that much and feels relief instead (Participant 2)

Just others to not feel sad and not give up (Participant 5) 
To not exaggerate... to not act as if this is the end of the world... (Participant 1)

What is more, half of the participants considered emotion regulation within the adolescent's environment to be helpful. An emotionally serene atmosphere wherein the all family members can manage stress and express painful feelings with a view to receive reassurance through fruitful discussion was also a desideratum.

... that tensions will be not... and others will not be stressed, because stress is transmittable, so when my parents feel anxious, I can sense it too (Participant 1)

... that I could talk to my parents or my sister about the problems that worry me (Participant 9)

... and they would tell me things like "this will go away soon", "it will not last too long, and then things will be like they used to be'(Participant 3)

A family's positive emotional state proved to be the most commonly reported helpful aspect for the majority of the adolescents. This in turn suggests that family would offer ample room for discussion as well as display empathy so that the children can feel a closeness with others and that they can be given support, escaping thus loneliness. In a similar vein, some participants reported the physical proximity to their beloved ones as quite a helpful parameter.

When, for example, my relatives discuss with me, this helps me a lot (Participant 6)

... and when, to a great extent, they understand how we feel (Participant 2)

What I need now, I believe, is for my family to stand by me... because I don't feel alone in that way (Participant 1)

It would help me very much, if the hospital staff could pressurise the institution staff to come and get me... as soon as possible... and then, if they could bring my brothers here to see them for a bit, but they can't actually consider where they live... (Participant 7)

Another helpful way of dealing with the coronavirus pandemic, as mentioned by two participants, was the stimulus for shared leisure time and even any welcome distractions from others.

... to spend our time creatively, to not have many moments that I would sit and think on my own about what will happen... so, when others are by me and draw my attention away from all this coronavirus issue, I believe this helps me a lot (Participant 1)

... when I discuss together with the people with whom I share the same space, this helps take our minds off all this (Participant 6)

One participant talked about future plans and the removal of restrictions as being a comforting expectation. 
... it helps for others to tell me several things we can do and arrange when the pandemic ends, for example, discussing where we can go out, or where to spend the summer holidays (Participant 6)

Another female reflected on her thoughts that people's compliance with the strict measures would help her feel better:

Maybe if all people would isolate themselves in their home, this would be helpful for me, because there are people who don't abide by the measures and go out and unfortunately that's how the virus is being transmitted (Participant 8)

Notably, the same participant reported that there is nothing that could help her:

I think there is nothing that could help me think even slightly differently about the coronavirus (Participant 8)

Her view chimes well with that of another participant:

No, I don't have anything... (Participant 4)

Two more adolescents could not think of any helpful practices at first; yet, shortly after they were soon able to pinpoint specific ways through which others could help them feel better.

\section{Discussion}

The aim of our research was to qualitatively investigate, through in-depth interviews, the perceptions, emotional reactions and needs of adolescent psychiatric inpatients amidst the COVID-19 pandemic. Preliminary knowledge about the complexity of subjective experience of this vulnerable category of adolescents, especially in the face of an unprecedented health crisis, will potentially enhance researchers', clinicians' and policy makers' understanding of the patients' perspective so that the former may develop tailored preventive and therapeutic interventions.

Regarding their knowledge about the impact of the current pandemic, almost all adolescents focused on information about the nature of coronavirus and on existing crisis management practices. Only one participant mentioned the probable psychosocial impact of the pandemic, and this may reflect a general trend to underestimate the ramifications of a mass disaster on mental health. Regarding changes to daily life, nearly all adolescent inpatients mostly identified negative changes due to the quarantine state, namely restrictions on social life and personal freedom as well as excessive contact with family members during home isolation. Considering that the subjects of the present study, were adolescents (thus, individuals in a critical developmental phase during which a sense of autonomy must be safeguarded by all accounts [25]), the deprivation and restriction of freedom must not be overlooked. According to the treatment plan followed in each case, a number of patients who were discharged from hospital and returned home due to the pandemic found home quarantine helpful, mainly because it provided them the opportunity to spend more time with their family. By contrast, other patients were 
ambivalent about this restriction. Interestingly enough, the patients who remained hospitalized during the pandemic focused on new hygiene rules and their contact with loved ones was decreased and restricted to family visits; some participants of this group reported no significant changes.

With regard to patients' feelings about the pandemic, they acknowledged anxiety about harm to self and beloved ones, mood changes within the family nucleus. Feelings of anxiety about the unknown, and the management of the pandemic in other countries were also manifested. Furthermore, sadness and grief about the deceased and the lack of contact with beloved ones were mentioned, while the feeling of entrapment due to the numerous restrictions yet another particularly interesting outcome. Concurrently, participants who were subjected to home isolation did acknowledge some positive feelings about spending more time with loved ones, yet ambivalent feelings were also recorded in this group of participants.

Concerning adolescents' helpful thoughts during the pandemic, our findings indicate that avoiding rumination about the coronavirus and its consequences, focusing on the positive, and looking towards the future were helpful strategies for coping with challenging feelings. Additionally, a sense of belongingness, provided that the adolescents in question were not alone and shared the commonality of experience instead, emerges as a key coping strategy. Trust in the work of authorities and the community was another particularly interesting point. Regarding helpful behaviors, both the adolescents who were discharged from hospital and some inpatients who remained hospitalized concurred that a balanced inflow and management of information, alongside the engagement in creative leisure activities and reaching out to others, were reported as beneficial. Concerning practices that enable adolescents deal with the pandemic, our study indicates adolescents benefit not only from receiving balanced health messages, but also from a sensible, balanced mindset within their social and family environment. Accordingly, emotional regulation of the environment was deemed by adolescents as a highly helpful practice. A positive emotional atmosphere within the familial setting was the most commonly reported helpful factor for the majority of adolescents, and the same applied for shared leisure time activity as well as the joint planning of future activities whilst applauding the individuals' adherence to the strict measures. However, some adolescents did not acknowledge any helpful external intervention, thus reflecting traces of a feeling of helplessness.

In our research, adolescents reported a wide array of negative thoughts and feelings which are pandemicrelated. Such thoughts/ feelings may be triggered either by the threat of the pandemic itself or by the changes incurred both the daily life and human relationships due to the pandemic or even by the reactions of either family members or the adolescent social support network. The negativity of thoughts and emotions may also have aggravated pre-existing adolescent mental health problems, even though it is also possible that the negativity also stems from the aforementioned mental health problem. That said, researchers and clinicians ought to investigate the pandemic-specific patient thoughts/ feelings and to focus on better understanding both the stressors and maintenance mechanisms. It is also vital that they shed further light on the role of pandemic-specific thoughts/feelings within the context of pre-existing mental disorders. 
Furthermore, adolescents mentioned various factors/ways that help them cope with stress-ridden experiences in the midst of the pandemic. Lending an ear to the patients' voices and taking into consideration their experience will enlighten the scientific community about the possible routes effective strategies to enhance patient resilience, and also prevent the aggravation of mental disorders whilst therapeutically addressing the patient needs during the pandemic.

Given all the aforementioned points, it would be feasible to argue in favor of the designing of personalized preventive and therapeutic interventions. Targets should be in accordance with the experience(s) of this extremely vulnerable population, and they should also facilitate sophisticated treatment planning decisions, such as staying hospitalized or sheltering at home. Given the fact that the role of the family and social environment in triggering, maintaining and managing difficulties during this crisis proved instrumental in the adolescents' responses, their inclusion in these interventions cannot be overstated.

Given the well-documented tendency to underestimate the impact of a mass disaster on mental health, it is essential for researchers to monitor and report symptoms of anxiety, depression and other mental health issues towards an enhanced understanding of the psychological pathways that mediate and also with a view to design and implement personalized interventions $[15,26]$. When it comes to hospitalized adolescents who are a priori considered a population already burdened by severe psychopathology, in particular, this is a serious wake-up call for researchers to address the issue of how it will be rendered possible to mitigate the mental health consequences of the vulnerable group in question in the midst of the pandemic, as well as on the impact of insatiable media consumption and health messaging surrounding COVID-19. Discovering, evaluating and ameliorating the interventions required to address the psychological, social, and neuroscientific aspects of the pandemic are also quintessential. [27, 28]. Furthermore, it is essential for clinicians who treat adolescents to engage in discourse with both the youth and the parent/guardian on the impact of the COVID-19 crisis on family life. Symptoms of depression and anxiety or hyperactivity in the adolescent may be situational and may recede either in time or with supportive intervention. Should major depression, anxiety disorders develop or should there is exacerbation of a current psychiatric disorder, medication and/or evidence-based psychotherapy will be required [29]. What is more, it is proposed that parents become actively involved in the entirety of the therapeutic design, not least because the mental health state of parents quite often deteriorates alongside the worsening of their children's behavioral health; in addition, there are cases where parents can address states of uncertainty and isolation while simultaneously helping their offspring cope with the new state of affairs due to COVID-19 [15, 16, 30, 31].

It should be noted here that the present research project offered insights by means of observing adolescents in two different types of treatment (inpatient and homebound) Remaining at home provided adolescents with further opportunities for interacting with others via technology. Indeed, such a practice was proved considerably useful for adolescents that received distance treatment. On the other hand, however, a number of the expected quarantine pitfalls [4] alongside social and physical distancing measures are also key risk factors for mental health issues (e.g. domestic and child abuse and 
psychosocial risks), thus requiring a thorough evaluation in the event of any modification of the therapeutic settings [27].

Lastly, the current study's findings highlight the contribution of the State's health policies in both imbuing and preserving a sense of security in the citizens. To this end, some suitable practices for both families and clinicians that will enable adolescents cope while in isolation may include: (a) establishing a regular routine and schedule at home, (b) helping adolescents keep in touch with friends and family members via technology, (c) keeping them informed about the current situation with honesty, using wording and concepts that are clearly intelligible, (d) helping adolescents find accurate and up-to-date information without overwhelming them, (e) developing a "shared understanding" within the family about coronavirus, and $(\mathrm{g})$ making future plans [11].

\section{Limitations}

The purpose of the present study was the collection of critical information on an unprecedented experience. It focused on a small sample of participants instead of seeking generalizable results. Consequently, the present findings do not aspire to reflect the whole range of perceptions and needs of the entire population. Moreover, future research should include in-depth interviews of parents and clinicians for the documentation of a variety of perspectives on youths' experiences.

\section{Conclusion}

Despite its limitations, the present study revealed important information about perceptions, feelings and thoughts of adolescent psychiatric inpatients during the COVID-19 pandemic. To our knowledge, there is still no other qualitative research dealing with adolescent psychiatric inpatients. The latter constitute a particularly vulnerable population with greater mental health risks than healthy adolescents. The information stemming from the present study may be proved useful for both researchers and decisionmakers so that interventions that effectively address children's mental health problems within the context of hospitalization during the pandemic can be successfully promoted. Despite the extremely challenging circumstances, professionals should steadfastly carry on with providing both standard and emergency mental health care in order to mitigate any negative consequences for children and adolescents alike. Continuity of mental health care during all phases of the pandemic can be facilitated by exhibiting flexibility and creativity.

\section{Declarations}

\section{Ethics approval and consent to participate}

The Bioethics Committee of the "Aghia Sophia" Children's Hospital approved this study protocol. Written consent from the parents and oral consent from the patients were obtained prior to starting the data collection. 
Consent to publication

Not applicable.

Availability of data and materials

The authors do not have the right to share any data information as per their institution policies.

\section{Competing interests}

The authors declare that they have no competing interests.

\section{Funding}

None.

\section{Authors' contributions}

GG designed the study; SM, AZ and GG carried out the analysis, interpreted the results and drafted the manuscript; MB, SC and GK assisted in reviewing the manuscript; GK supervised the study; All authors reviewed the final manuscript and gave their consent.

\section{Acknowledgments}

The authors would like to thank all participants who helped us during this project. Our gratitude also goes to Mrs. Iris Vamvaka for her assistance with language editing.

\section{References}

1. Flahault A: COVID-19 cacophony: is there any orchestra conductor? Lancet 2020, 395(10229):1037.

2. Laor N, Wolmer L: Children exposed to mass emergency disaster: the role of the mental health professionals. In: Lewis's child and adolescent psychiatry: a comprehensive textbook. 5th edn. Edited by Martin A, Bloch MH, Volkmar FR. Philadelphia: Wolters Kluwer; 2018: 1890-1927.

3. Bao Y, Sun Y, Meng S, Shi J, Lu L: 2019-nCoV epidemic: address mental health care to empower society. Lancet 2020, 395(10224):e37-e38.

4. Brooks SK, Webster RK, Smith LE, Woodland L, Wessely S, Greenberg N, Rubin GJ: The psychological impact of quarantine and how to reduce it: rapid review of the evidence. Lancet 2020, 395(10227):912-920.

5. Lima CKT, Carvalho PMM, Lima I, Nunes J, Saraiva JS, de Souza RI, da Silva CGL, Neto MLR: The emotional impact of Coronavirus 2019-nCoV (new Coronavirus disease). Psychiatry Res 2020, 287:112915.

6. Shigemura J, Ursano RJ, Morganstein JC, Kurosawa M, Benedek DM: Public responses to the novel 2019 coronavirus (2019-nCoV) in Japan: Mental health consequences and target populations. In: 
Psychiatry Clin Neurosci. Volume 74, edn.; 2020: 281-282.

7. Xiang YT, Yang Y, Li W, Zhang L, Zhang Q, Cheung T, Ng CH: Timely mental health care for the 2019 novel coronavirus outbreak is urgently needed. Lancet Psychiatry 2020, 7(3):228-229.

8. Fitzpatrick KM, Harris C, Drawve G: Fear of COVID-19 and the mental health consequences in America. Psychol Trauma 2020, 12(S1):S17-s21.

9. Pierce M, Hope H, Ford T, Hatch S, Hotopf M, John A, Kontopantelis E, Webb R, Wessely S, McManus $S$ et al: Mental health before and during the COVID-19 pandemic: a longitudinal probability sample survey of the UK population. Lancet Psychiatry 2020.

10. Loades ME, Chatburn E, Higson-Sweeney N, Reynolds S, Shafran R, Brigden A, Linney C, McManus MN, Borwick C, Crawley E: Rapid Systematic Review: The Impact of Social Isolation and Loneliness on the Mental Health of Children and Adolescents in the Context of COVID-19. J Am Acad Child Adolesc Psychiatry 2020.

11. Singh S, Roy D, Sinha K, Parveen S, Sharma G, Joshi G: Impact of COVID-19 and lockdown on mental health of children and adolescents: A narrative review with recommendations. Psychiatry Res 2020, 293:113429.

12. Duan L, Shao X, Wang Y, Huang Y, Miao J, Yang X, Zhu G: An investigation of mental health status of children and adolescents in china during the outbreak of COVID-19. J Affect Disord 2020, 275:112118.

13. Liu JJ, Bao Y, Huang X, Shi J, Lu L: Mental health considerations for children quarantined because of COVID-19. Lancet Child Adolesc Health 2020, 4(5):347-349.

14. Ghosh R, Dubey MJ, Chatterjee S, Dubey S: Impact of COVID -19 on children: special focus on the psychosocial aspect. Minerva Pediatr 2020, 72(3):226-235.

15. Fegert JM, Vitiello B, Plener PL, Clemens V: Challenges and burden of the Coronavirus 2019 (COVID19) pandemic for child and adolescent mental health: a narrative review to highlight clinical and research needs in the acute phase and the long return to normality. Child Adolesc Psychiatry Ment Health 2020, 14:20.

16. Patrick SW, Henkhaus LE, Zickafoose JS, Lovell K, Halvorson A, Loch S, Letterie M, Davis MM: Wellbeing of Parents and Children During the COVID-19 Pandemic: A National Survey. Pediatrics 2020.

17. Moreno C, Wykes T, Galderisi S, Nordentoft M, Crossley N, Jones N, Cannon M, Correll CU, Byrne L, Carr $S$ et al: How mental health care should change as a consequence of the COVID-19 pandemic. Lancet Psychiatry 2020, 7(9):813-824.

18. Sheridan Rains L, Johnson S, Barnett P, Steare T, Needle JJ, Carr S, Lever Taylor B, Bentivegna F, Edbrooke-Childs J, Scott HR et al: Early impacts of the COVID-19 pandemic on mental health care and on people with mental health conditions: framework synthesis of international experiences and responses. Soc Psychiatry Psychiatr Epidemiol 2020:1-12.

19. Cui Y, Li Y, Zheng Y, Chinese Society of C, Adolescent P: Mental health services for children in China during the COVID-19 pandemic: results of an expert-based national survey among child and adolescent psychiatric hospitals. In: Eur Child Adolesc Psychiatry. Volume 29, edn.; 2020: 743-748. 
20. Compas BE, Jaser SS, Bettis AH, Watson KH, Gruhn MA, Dunbar JP, Williams E, Thigpen JC: Coping, emotion regulation, and psychopathology in childhood and adolescence: A meta-analysis and narrative review. Psychol Bull 2017, 143(9):939-991.

21. Jefsen OH, Rohde C, Nørremark B, Østergaard SD: Editorial Perspective: COVID-19 pandemic-related psychopathology in children and adolescents with mental illness. J Child Psychol Psychiatry 2020.

22. Aishworiya R, Kang YQ: Including Children with Developmental Disabilities in the Equation During this COVID-19 Pandemic. J Autism Dev Disord 2020:1-4.

23. Given L: The SAGE Encyclopedia of Qualitative Research Methods. Los Angeles, CA: Sage Publications, Inc; 2008.

24. Morse J, Richards L: Read Me First for a User's Guide to Qualitative Methods. Thousand Oaks, CA: Sage Publications, Inc; 2002.

25. Christie D, Viner R: Adolescent development. Bmj 2005, 330(7486):301-304.

26. Golberstein E, Wen H, Miller BF: Coronavirus Disease 2019 (COVID-19) and Mental Health for Children and Adolescents. JAMA Pediatr 2020.

27. Holmes EA, O'Connor RC, Perry VH, Tracey I, Wessely S, Arseneault L, Ballard C, Christensen H, Cohen Silver R, Everall I et al: Multidisciplinary research priorities for the COVID-19 pandemic: a call for action for mental health science. Lancet Psychiatry 2020, 7(6):547-560.

28. Wade $M$, Prime $H$, Browne DT: Why we need longitudinal mental health research with children and youth during (and after) the COVID-19 pandemic. Psychiatry Res 2020, 290:113143.

29. Wagner KD: Addressing the Experience of Children and Adolescents During the COVID-19 Pandemic. J Clin Psychiatry 2020, 81(3).

30. Russell BS, Hutchison M, Tambling R, Tomkunas AJ, Horton AL: Initial Challenges of Caregiving During COVID-19: Caregiver Burden, Mental Health, and the Parent-Child Relationship. Child Psychiatry Hum Dev 2020:1-12.

31. Spinelli M, Lionetti F, Pastore M, Fasolo M: Parents' Stress and Children's Psychological Problems in Families Facing the COVID-19 Outbreak in Italy. Front Psycho/ 2020, 11:1713.

\section{Tables}

Table 1 Knowledge about the pandemic

Conceptual category 1: Information about the nature of the coronavirus

Concepts

Definition of the coronavirus

Worldwide spread rate 
Morbidity

Transmission risk/ rate

Mortality

Outcome

Previous knowledge about the pandemic

Origins of the virus

Conceptual category 2: Combating coronavirus pandemic

Concepts

Existing crisis management practices in Greece

Existing knowledge about treatment and disease prevention

Conceptual category 3: Psychosocial aftermath of the pandemic

Concepts

Psychosocial consequences

Table 2 Changes in everyday life

Conceptual category 1: Positive changes in everyday life

Concepts

Precautionary measures

Increased contact with beloved ones

Conceptual category 2: Negative changes in everyday life

Concepts

New obligatory protective behaviors 
Social life restraint

Restriction of freedom and prohibitions

Increased contact with beloved ones

Conceptual category 3: Non-important changes

Concepts

Little change

Table 3 Feelings about the pandemic

Conceptual category 1: Negative feelings

Concepts

Worries about possible harm to oved ones

Worries about possible harm to self

Worries about the future and the unknown

Concerns about the management of the pandemic from a global perspective

Sadness about the deceased or affected people

Sorrow with regard to the lack of contact with beloved ones

Feeling confined or being deprived of their freedom

Conceptual category 2: Positive feelings

Concepts

Optimism

Joy because of the increased time spent with beloved ones

Conceptual category 3: Ambivalence

Concepts

Mixed feelings

Conceptual category 4: Non-important changes

Concepts

Little change 
Table 4 Helpful thoughts and behaviors

Conceptual category 1: Helpful thoughts

Concepts

Avoiding rumination about the pandemic

Focusing on the positive aspects of the current situation

Looking into the future

Shared experience - feeling that they are not alone

Confidence in authorities and community

Conceptual category 2: Helpful behaviors

Concepts

Calibrating the amount of incoming information

Creative leisure activities

Relating to others

Table 5 Practices of helping adolescents deal with the pandemic

Conceptual category 1: Balanced health messaging

Concepts

Calibrating the provision of information

Answering adolescents' queries

Hearing positive updates

Conceptual category 2: Balanced approach and mode of thinking in the environment

Concepts

Family's positive attitude

Avoidance of excessive panic

Conceptual category 3: Emotion regulation within the environment

Concepts

Calm emotional climate

Family's ability to manage their own stress

Having the chance to express painful feelings

Receiving reassurance through discussion 
Conceptual category 4: Family's positive emotional climate

\section{Concepts}

Providing opportunities for discussion

Showing empathy

Feeling close to others, avoiding loneliness

Physical proximity

Conceptual category 5: Shared leisure time and distraction

Concepts

Shared leisure time and distractions coming from others

Conceptual category 6: Looking into the future

Concepts

Future plans

Future removal of restrictions

Conceptual category 7: Compliance

Concepts

Compliance with the strict measures

Conceptual category 8: Nothing

Concepts

Nothing could help 